10

\title{
Graphical Means of Directing Users' Attention in the Visual Interface
}

\author{
Shumin Zhai Julie Wright Ted Selker Sabra-Anne Kelin \\ IBM Almaden Research Center \\ 650 Harry Road, MS NWE/B2 \\ San Jose, CA 95120 USA \\ \{zhai, amethyst, selker\}@almaden.ibm.com, sakelin@mit.edu
}

\begin{abstract}
This paper presents two experiments in using "masks" to direct users' attention within a graphical user interface. Bleaching, darkening, blurring, and a solid-color pattern overlay (screening) are used to de-emphasize background material, causing the target to visually "pop-out" at the user. The tradeoff between effectively directing the user's attention and ensuring the readability of the background material is explored. Experimental results indicate that there is a wide range of darkening and screening levels that can create a pop-out effect without degrading the readability of the masked area.
\end{abstract}

KEYWORDS attention, pop-out effect, help systems, visual masking, user interface techniques

\section{INTRODUCTION}

Computers can present users with a seemingly endless variety of options and data. This complexity, which can easily overload users, has lead to the use of techniques such as 'wizards', simplified, step-by-step interfaces that break up tasks into small, easy-to-digest parts. This simplicity, however, is often gained at the expense of the flexibility and efficiency more traditional interfaces can provide. For instance, a wizard often locks the user into a particular sequence of choices that may not be suitable for a particular user. The tradeoff between simplicity and flexibility/ efficiency suggests a third interface approach that combines both the simplicity of wizards and the flexibility and efficiency of a more traditional interface. One such an approach is illustrated by the Cognitive Adaptive Help (COACH) agent system, which annotates traditional interfaces for inexperienced users (Selker, 1994). In $\mathrm{COACH}$, dialogs are partially covered with a bitmap (mask). Only one step in the task remains uncovered at a time, in order to focus the user's attentions on this single step. At each step,
$\mathrm{COACH}$ provides help and navigational control information in an external bubble.

Directing a user's attention by masking (de-emphasizing) parts of the visual display also has applications beyond help or tutor systems. Many examples exist in mainstream applications, such as Lotus Word Pro and Microsoft Windows95. When a user chooses to quit Microsoft Windows95, the desktop is covered with a pattern of black dots, making it appear darker than the confirmation dialog. In Word Pro, the brightness of colors on inactive icons or controls is reduced, causing them to look washed-out.

Masked objects may retain their readability so as to provide orientation, access to obscure or advanced options, or recall of previous settings. Yet by creating perceptual distance between the highlighted (unmasked) and de-emphasized (masked) areas, it is possible to direct the user's visual attention to the emphasized area.

The process of highlighting information is a significant part of traditional graphic design. Underlining, changing font, changing color, and changing the background are commonly used in printed

Human-Computer Interaction: INTERACT'97 S. Howard, J. Hammond \& G. Lindgaard (editors)

Published by Chapman \& Hall OIFIP 1997 
media. Many such visual dimensions that can produce a "pop out" effect. One candidate dimension is color. However, a color's pop-out effect is complicated. For example, an orange dot will stand out amidst a mass of yellow dots. An orange dot will also stand out amidst many red dots, but the same orange dot will not stand out among a collection of both yellow and red dots (D'Zmura, 1991). Consider also a 460-nm (wavelength) spot. Such a spot will usually appear blue. But, if that same spot is made very small, it will look white. Brightness is also a factor. The perceived brightness of a region depends heavily on the brightness of the surrounding area. A lighter background tends to darken, while a darker background tends to lighten. Color, luminance, contrast, area, spatial frequency, and temporal considerations are among the factors that interact to determine visual perception (Rogowitz, 1983). Most importantly, color is also a dimension that application designers rely heavily upon. Using color to produce a pop out effect in a computer interface is likely to interfere with the application's use of color.

Groupings of many small elements can form textures that also have a pop-out effect. For example, a vertical bar will stand out in a field of horizontal bars (D'Zmura, 1991). Elements of different size or complexity, such as a group of plus signs ('+') randomly oriented in a field of randomly oriented minus signs ('-') will also pop-out, but the same phenomenon will not work using a ' $T$ and an ' $L$ ' (Julesz, et al., 1983). Using texture to produce a 'pop-out' effect may also invade or restrict an application designer's visual design space.

Instead of using color or texture that invade an application designer's design space, the techniques explored in the following experiments manipulate brightness, contrast, and resolution to produce a perceptual difference between what is masked and what is unmasked. We were interested in testing if these approaches would produce effective pop-out effect without damaging the legibility of the masked objects.

\section{EXPERIMENT 1}

\subsection{Design}

Three masking techniques, namely darkening, bleaching, and blurring, were used in this experiment. Each has a metaphor grounded in everyday experience. Darkening creates the effect of a spotlight on a darkened stage. Bleaching makes the masked area look faded, possibly causing the bright, vivid colors in the unmasked area to stand out. Blurring was an attempt to mimic a person's vision system: only the area being focused on is received in high acuity, but the objects in the periphery are in low resolution. We hoped the user's attention would be automatically drawn to the unblurred object.

An experiment was designed to test if these techniques were indeed effective. Adobe Photoshop was used to produce the masked image. To darken the masked areas, the brightness was reduced $(-100)$, while the contrast was increased $(+35)$. Bleached masking was created by reducing the contrast $(-60)$. A Gaussian filtering of 1.5 pixels was applied to create the blurred condition.

In the experimental task, a dialog was created containing over 40 common interface items (buttons, radio buttons, check-boxes, entry fields, and so forth, see Figure 1 and 2). For each mask condition, subjects

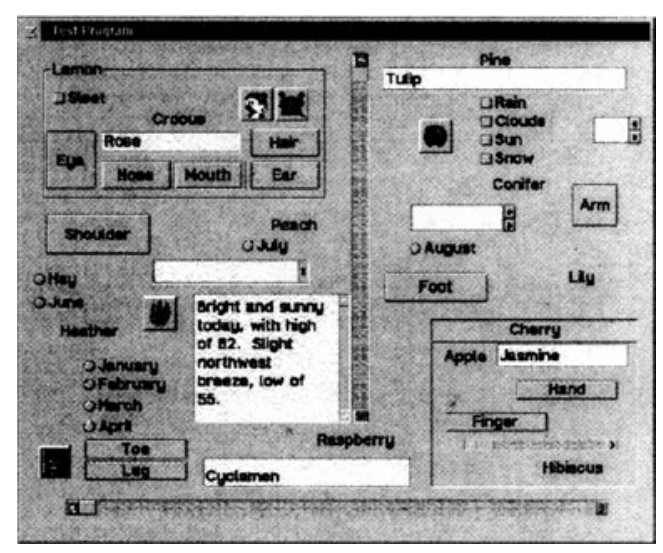

Figure 1: Dialog for Experiment 1 before masking

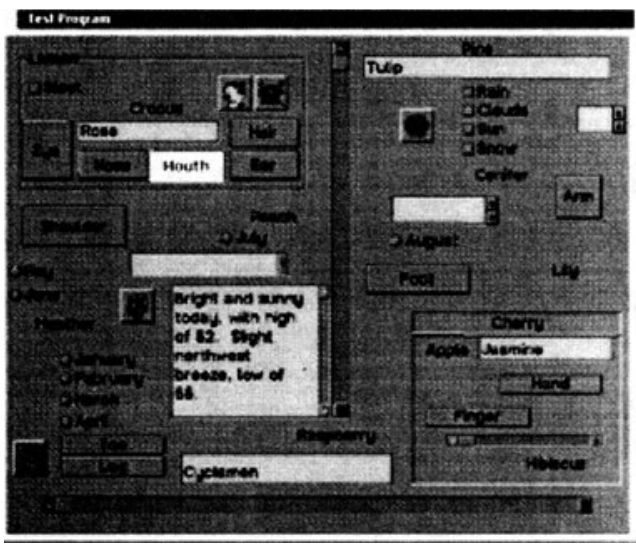

Figure 2: The same dialog after masking (by darkening) all objects but the button 'mouth' 
Selection Times

With Standard Error error bars.

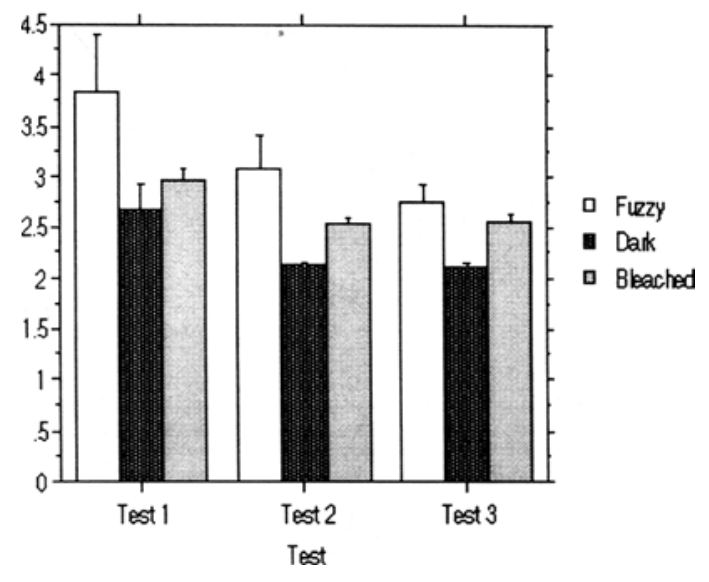

Figure 3: Response times (seconds) for experiment 1

were shown the dialog with the mask applied everywhere but on the target item (Figure 2). Subjects were instructed to find the target that stood out and to click it as quickly as possible. The dialog would disappear, then reappear with a different target item unmasked. Three tests were conducted for each masking technique. Each test consisted of 20 target selections. The testing order of the three masking techniques was balanced in a Latin square pattern. A total of 18 subjects participated in the experiment. A questionnaire, filled out at the end of the experiment, asked subjects to rate the three masking techniques according to the ease of finding the target and the readability of the masked portion of the dialog.

\subsection{Results}

Repeated measure analysis of variance (ANOVA) on the selection time data showed that the blurring technique was significantly inferior to the darkening technique $\left(F_{1,15}=6.5 . p<0.05\right)$. Other pair comparisons were insignificant. Subjects' performance improved through practice (from early tests to later tests, see Figure 3) significantly $\left(F_{2,130}=7.3 . p<0.01\right)$, but such a factor did not interact with the techniques. Neither experimental order, nor target type (pictures, words and others) nor any interaction was significant.

As measured by subjective rating data, all pairs of techniques were significantly different from each other, both for target selection $(p<0.05)$ and for readability of background information $(\mathrm{p}<0.001)$ (see Figure 4).

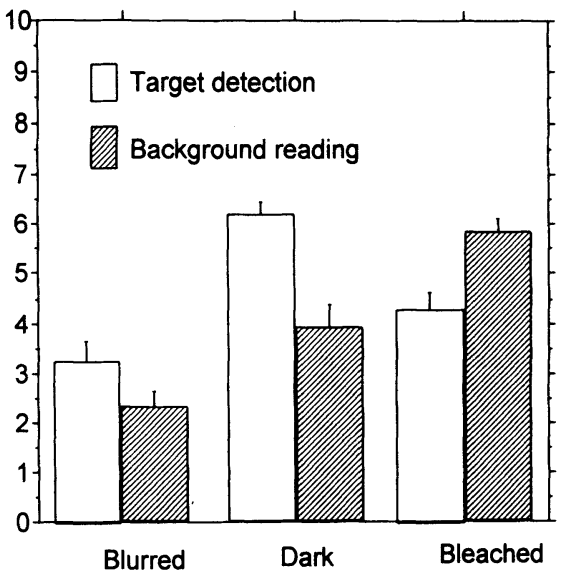

Figure 4: Subjective rating in Experiment 1 $(10=$ very easy, $0=$ very difficult $)$

Based upon the performance measurements and the subjective ratings, the blurring technique appeared to be inferior to the other two techniques, both for target selection and background readability. The difference between bleaching and darkening was less clear. Darkening appeared to be better for target pop-out effect; bleaching better for maintaining background readability (Figure 4).

This experiment indicated that the design of a mask could affect both performance and preference, and that an interesting design (such as the blurring technique) does not necessarily produce good results. It is also informative to note that the target type (either icon, words or others) did not change the masking effect.

There are a number of issues that were not resolved by this experiment. First, while the fact that the subjects were able to find the unmasked target (they were asked to click objects that stand out, no additional description of the targets was given) showed that all of the masking techniques effectively directed the user to the targets and two of the techniques were more effective than the other, it was unclear how strong such an effect was in comparison to searching for a target in the absence of a mask. Second, the experiment's assessment of the readability of the masked area was supported only by subjective data. Third, we suspected that the gain in making a target stand out might sacrifice the readability of the masked area. This would certainly be true if masking was taken to the extreme. Consider, for example, the most extreme level of the darkening technique. The background area would be darkened until it turned solid black. This would 


\begin{tabular}{|c|c|c|c|c|c|c|}
\hline COAL & COAL & COAL & COAL & & & Darken \\
\hline COAL & COAL & COAL & $\mathrm{COAL}$ & COAL & & Bleached \\
\hline COAL & COAL & COAL & COAL & COALL & 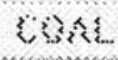 & Screened \\
\hline No Mask & Level 1 & Level 2 & Level 3 & Level 4 & Level 5 & \\
\hline
\end{tabular}

Figure 5: Sample buttons for each mask and density in Experiment 2

certainly cause the target item to stand out, but items in the masked area would be completely hidden. The tradeoff between the pop-out effect and the readability of the masked area might be a function of the masking density: the heavier the masking is, the stronger the pop-out effect might be, but the poorer the readability of the masked area could become (See Figure 5). Is this a linear tradeoff, or is there, as in the design of partially transparent menus (Harrison, et al., 1996), a range of masking density that provides both a pop-out effect and readability of the masked objects? A second experiment was designed to address these issues.

\section{EXPERIMENT 2}

\subsection{Design}

Four masking conditions were tested: bleaching, darkening, screening, and no-masking (see Figure 5 for the samples used). The bleaching and darkening masks were similar to those used in the first experiment. The addition of the screening technique, which overlaid a uniform, periodic, yellow pattern onto the masked areas, was motivated by its low cost of implementation: only a percentage of pixels have to be replaced with yellow color. The blurred mask was not tested because it did not perform well in the first experiment. The "no mask" condition was used as a control condition.

Experiment 1 only tested one level of masking for each technique. This experiment tested five levels (from 1 to 5) of masking with for each technique were tested. The five levels of bleaching were created by reducing the contrast $(-30,-45,-60,-75,-95)$. The five levels of darkening were created by increasing the contrast $(+50)$ and decreasing brightness $(-20,-40,-60$, $-80,-100)$. The levels of screening were created by replacing a pattern of pixels with yellow pixels (RGB $25525585)$ such that a percentage of the pixels were blocked out $(12 \%, 25 \%, 37 \%, 62 \%, 75 \%)$. Note that the intervals within each technique were not necessarily equal. The values were chosen and adjusted to cover as wide as possible a range of appearance (see Figure 5) according the authors' subjective experience and pilot testing. The lowest levels of masking appeared sufficiently close to no-mask condition and the highest levels of masking made the masked words nearly indistinguishable.

A target matching task was designed for this experiment. Subjects were instructed to select, as quickly as possible, the button that matches the target word at the top of the dialog as shown in Figure 6. The advantage of this experimental scenario, in comparison with Experiment 1 was that all three aspects of masking could be evaluated. First, subjects' performance of searching the target word when no mask was applied served as the normal, control condition. Such a normal condition can also be considered as Level 0 masking with any of the three technique. Second, when the target word was highlighted by various levels of masks applied to other words, the pop-out effect could be measured in comparison to the control condition. Third, when the target word was masked together with all other buttons, the readability of the masked area could be evaluated.

A total of 13 subjects performed the task under the normal control condition as well as all five levels of all three masking techniques. For each level in each masking technique, two sets of trials were run. In one set of trials, the target word was left unmasked (therefore highlighted) while the rest of the buttons

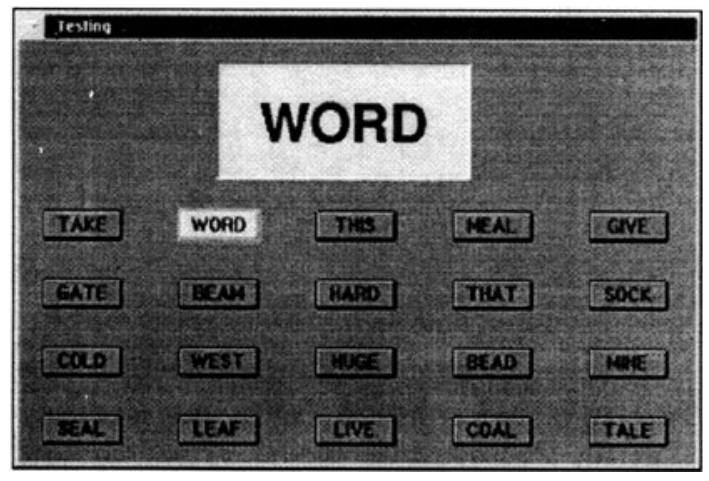

Figure 6: Test dialog for Experiment 2 (in darkening condition) 


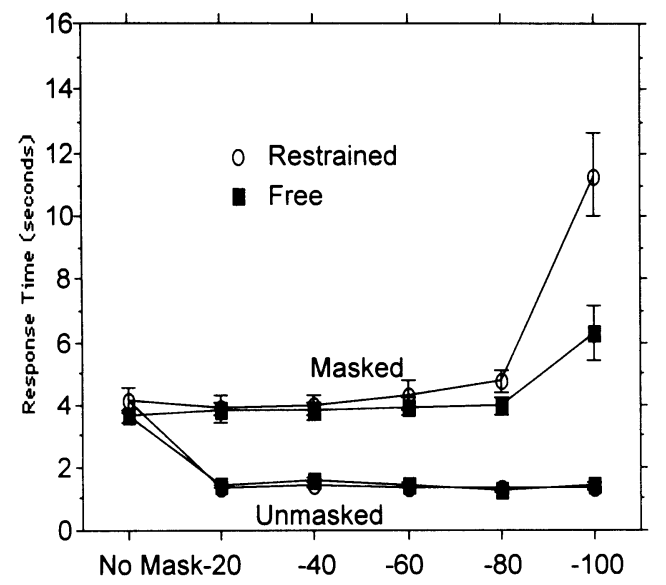

Figure 7: Mean task completion times and standard errors when the target was (top curves) masked or (bottom curves) highlighted / unmasked using the darkening technique.

were masked. In the second set, the entire dialog was masked, including the target word.

Each set of trials consisted of 5 practice trials followed by 20 test trials. Each of the test trials in a set corresponded to one of the 20 words (buttons) in the dialog (Figure 6), so all sets of trials encompassed the equal total travel distances. The order of the buttons was changed randomly between sets to avoid memorization of a button's placement.

The order of the masking conditions was determined by a Latin-square sequence of the darkening, bleaching, and screening techniques. The normal control condition was randomly inserted into the Latin-square sequence. For a given masking condition, the order in which the densities were tested was random.

During pilot testing, subjects tended to move closer to the computer screen under the more difficult conditions, presumably to compensate for increased difficulty. It seemed possible that subjects could maintain the same performance by exerting extra effort in more difficult conditions. A between-subject factor was introduced to explore this possibility. Six of the subjects were allowed to move their head position freely and seven subjects were prevented from moving closer than 1.5 feet by a string across the chest.

\subsection{Results}

\section{Masking by Darkening}

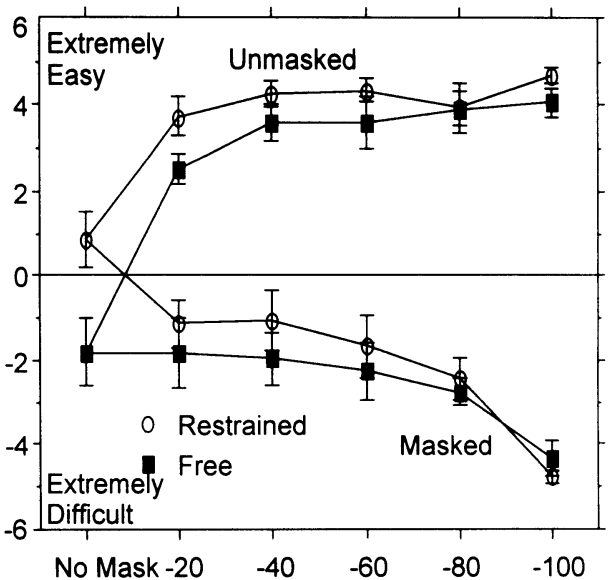

Figure 8: Subjective ratings with the darkening technique. +5 is extremely easy and -5 is extremely difficult.

Figure 7 illustrates the performance results of the darkening technique. First, let us examine the pop-out effect when the target was the highlighted/unmasked word. The bottom curves showed that, in comparison to Level 0 (no masking), selection time was significantly reduced when the rest of window was masked (darkened) to each of the masked levels: $F_{5,55}=110.9$, $\mathrm{p}<.0001$, once again showing that darkening is an effective means of creating a pop-out effect. Interestingly, even the slightest level of darkening tested appeared to make the unmasked object "pop out": mean selection time changed from 3.9 seconds at no darkening to 1.4 second at Level 1 (-20) darkening. It appeared that even the lowest level of masking enabled the subjects to be "cued" to, instead of searching for, the target. Further darkening did not make subjects perform much faster: mean selection time changed from 1.41 seconds at Level 1 to 1.36 second at Level 5. Such a difference was not statistically significant. Subjective rating data, however, appeared to be more sensitive to the darkening level increase (Figure 8). The higher levels of darkening appeared to make the unmasked target more distinguishable and were rated significantly easier than the lower levels of darkening. For example, a $T$-test showed $\mathrm{p}<.0001$ significance between Level 1 $(-20)$ and $5(-100)$.

Second, as shown by the top curves in Figure 7, subjects' performance in reading the masked area was not significantly affected by darkening level except at the very extreme (Level 5). T-tests showed that selection in the masked area at the highest level $(-100)$ 
was significantly slower than the other levels $(p<$ .0001 ), but the differences among other levels tested were relatively small (from 3.9 seconds at Level 0 to 4.4 seconds at Level 4 , statistically insignificant). It is remarkable that subjects maintained similar performance for such a wide range of apparent difficulty (See Figure 5). Subjective rating scores on selecting masked targets moved towards "extremely difficult" as darkening level increased (Figure 8). Not only was highest level $(-100)$ rated significantly more difficult than other levels $(p<0.0001)$, but levels 3 and 4 were also rated more difficult than Level 0 (no darkening): $\mathrm{p}<0.05$.

Viewing condition (Restrained vs. Free moving) was weakly significant to selection time in masked area: $F_{1,11}=4.4, p=0.06$. By moving closer to the screen as one strategy to compensate the increasing difficulty, the free moving group had faster selection time only at the very dark levels. There was a strong interaction between viewing condition and darkening level $\left(F_{5,55}=8.6, p<0.0001\right)$ in selecting masked targets. This was the only case in all performance and subjective data with all masking techniques where viewing condition made a statistically significant difference in the experiment.

To summarize, the results showed that except at the very extreme, a wide range of darkening levels can create a pop-out effect without significantly damaging the readability of the masked objects, although as the darkening level increases users may subjectively experience a slight increase in pop-out effect and a slight decrease in readability (compare Figure 7 and 8). Subjects tended to maintain the same level of performance by exerting additional effort, as indicated by the smaller time increase in selecting masked targets by the free moving group (Figure 7).

\section{Masking by Bleaching}

As shown in Figure 9 (bottom curves), the lowest level $(-30)$ of bleaching tested did not appear to be effective enough to direct the subjects' attention. Although it caused significantly shorter selection time than without masking (mean time 3.89 vs. $3.33, \mathrm{p}<$ .005 ), stronger bleaching produced still shorter selection time: $p<.0001$. This suggests that Level 1 bleaching used in the experiment did not produce the pop-out effect needed to fully utilize viewers' preattentive perception. For the rest of the bleaching levels, mean selection times were (from Level 2 to Level 5) 1.65, 1.33, 1.38, 1.39 seconds, no significant difference was found among these means. Subjective rating indicated that as bleaching level increased,

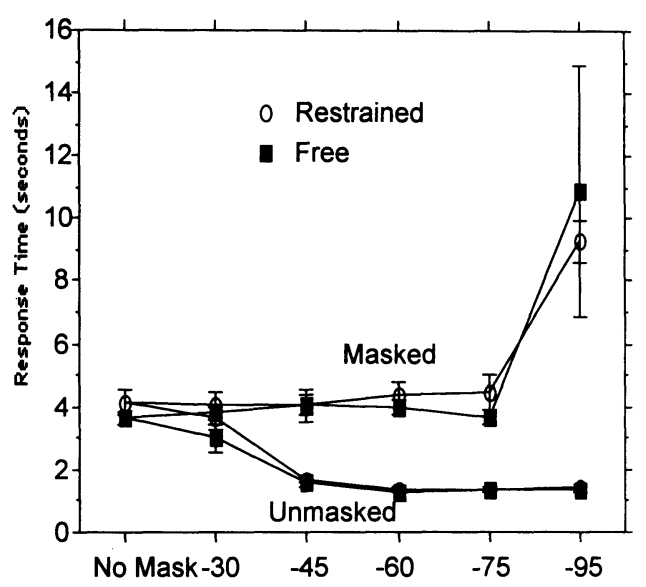

Figure 9: Task completion times when the target was (top curves) masked or (bottom curves) highlighted / unmasked using the bleaching technique.

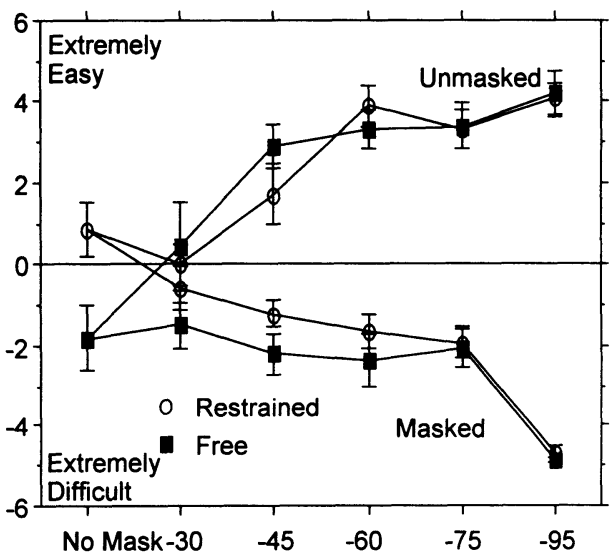

Figure 10: Subjective ratings with the bleaching technique. +5 is "extremely easy" and -5 is "extremely difficult".

selecting the unmasked target became significantly easier (see Figure 10).

The readability of the bleached area was similar to that of the area masked by darkening: selection time at the very extreme (-95) was significantly different from the rest $(p<0.0001)$. Differences among Level 0 (no mask) to Level $4(-75)$ were not significant, although subjective rating indicated increased difficulty.

Overall, in comparison to the darkening technique, a much narrower range of bleaching levels can accommodate both the pop-out effect and readability (compare Figure 9 against 7, Figure 10 against 8), although the best performances achieved by the 
bleaching technique were similar to those achieved by the darkening technique (about 4 seconds for selection in masked area and 1.4 seconds for selecting unmasked target).

\section{Masking by screening}

As shown in Figure 11, even the slightest level of screening tested (Level 1, 12\% pixels screened out) appeared to provide a strong cue: mean selection time of unmasked targets changed from 3.9 seconds at Level 0 screening to 1.5 second at Level 1 screening ( $p<$ 0.0001 ). Further screening did not appear to be much more effective; mean selection time changed from 1.5 seconds at Level 1 to 1.38 seconds at Level 5 (not statistically significant). Subjectively, even the lowest level of screening (level 1) made the task significantly easier $(p<0.0001)$. Further increase in screening level made the task seem even easier $(p<0.05)$ (Figure 12).

As the percentage of pixels masked out increased, the readability of the masked area was expected to decrease. Indeed, T-tests showed significantly different ratings from lower levels to higher levels (e.g. $p<0.05$ from Level 2 to Level 4) (see Figure 12). However, the time of selecting the target word from the masked area remained flat except at the extreme Level 5 (Figure 11). From Level 0 to Level 4, mean selection time changed from 3.9 to 4.1 seconds (not statistically significant). Only Level 5 was significantly different from the rest $(p<0.0001)$. This once again showed users' ability to overcome increased difficulty without significantly reducing time performance.

Overall, a wide range of screening, from $12 \%$ of pixels to $62 \%$ pixels being masked out, produced the optimal performance (again 4 seconds for selection in masked area and 1.4 seconds for selecting unmasked target).

\section{DISCUSSION}

All three techniques used in the second experiment were effective when adequate levels of masking were selected. The highlighted target selection time at such levels was around 1.4 seconds for all three techniques, much faster than with no masking (around 4 seconds). Bear in mind that motor control (moving cursor to target) was involved in target selection. The visual difference between finding a pop-out target and searching for the same target without masking cues was much greater than 4.0:1.4, the ratio of response times for the two conditions. Furthermore, for several levels of each mask, the time to find an item in the masked area was similar to Level 0 (no mask), suggesting that the readability of the masked areas had been well preserved.

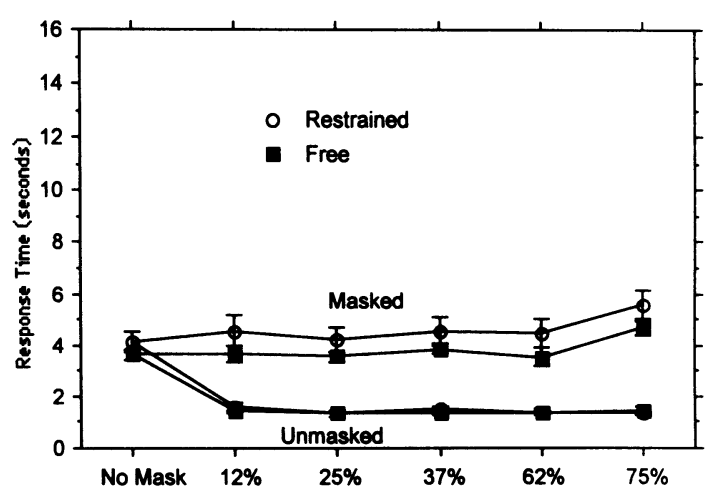

Figure 11: Task completion times when the target was (top curves) masked or (bottom curves) highlighted / unmasked using the screening technique.

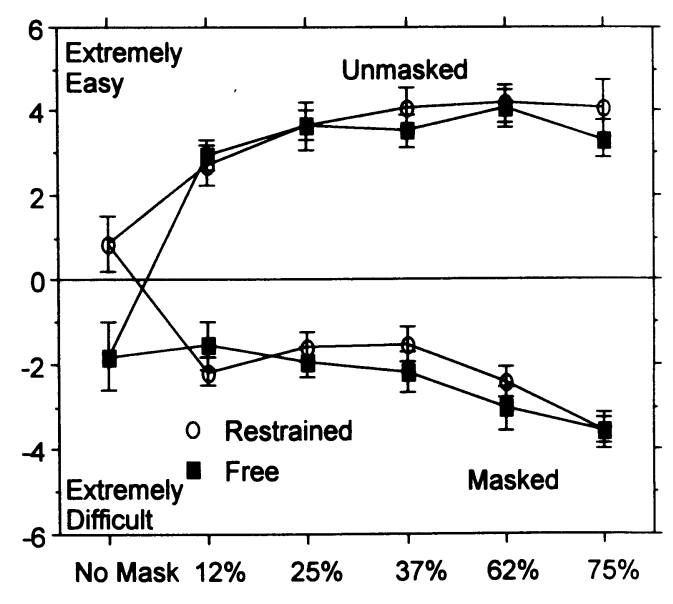

Figure 12: Subjective ratings with the screening technique. Horizontal axis is the degree of screening. +5 is "extremely easy" and -5 is "extremely difficult".

The range of such adequate masking levels that can provide strong cues to direct users' attention without readability loss in the masked area differed among the three techniques. The flat area of the performance curve where performance did not differ significantly in this experiment was -20 to -80 with the darkening technique, -45 to -75 with the bleaching technique, and $12 \%$ to $62 \%$ for the screening condition. Bleaching provided a narrower optimal range than the other two techniques. Darkening and screening both worked well, although they offer very different masking appearances and require different implementation mechanisms.

Although subjective data tend to vary from individual to individual, the rating data collected in the experiments did offer information not revealed by the 
performance data. The rated difficulty changed faster than the performance measurement as masking level moved to the two extrema. It is interesting that this increase in subjective difficulty did not correspond to immediate performance loss. Subjects tended to compensate for the increased difficulty, perhaps by exerting extra effort. See (Wickens, 1992) for related theories of performance and resources.

Note also that the experimental scenario is simpler than a typical application where both the visual features and the user's cognitive activities are much more complex. A narrowed range of effective masking level is expected in more complex applications.

\section{CONCLUSION}

Two experiments were conducted to evaluate means of drawing user's attention to various parts of a computer dialog. Four masking techniques were studied. One of them, blurring, did not appear to be as effective as the rest. Two techniques, darkening and screening, can provide a wide range of masking levels that could produce strong pre-attentive visual cues to pop-out the targets and still allow the user to read the masked area. Masks with as little as $12 \%$ pixels being screened out could produce a strong pop-out effect.

The studies presented here indicated that important information can be made to pop-out using darkening or screening masks. Darkening or screening the less relevant parts of the user interface provides strong attention cues to the important information. Yet the non-important information can still be accessed. Such a way of directing a user's attention by masking is a graceful human-machine communication method: telling the user what the user interface agent "thinks" is important, while allowing the user act according to his own will.

\section{ACKNOWLEDGMENTS}

We would like to thank Ron Barber, Paul Maglio and other members of the USER (User System Ergonomics Research) group at the IBM Almaden Research Center for their contributions to the development of this research.

\section{REFERENCES}

D'Zmura, M. Color in Visual Search. Vision Research, 31, 6 (1991), 951-966.

Harrison, B., and Vicente, K. An Experimental Evaluation of Transparent Menu Usage. Proceedings of CHI 96: Human Factors in Computing Systems (1996), 391-398.

Julesz, B., and Bergen, J. R. Textons, the Fundamental Elements in Preattentive Vision and Perception of Textures. The Bell System Technical Journal, 62, 6 (July-August, 1983), 1619-1645.

Rogowitz, B. E. The Human Visual System: A Guide for the Display Technologist. Proceedings of the SID, 24, 3 (1983), 235-252.

Selker, T. COACH: A teaching agent that learns. Communications of the ACM 37, 7 (1994), 92-99.

Wickens, C. D. Engineering Psychology and Human Performance, Second Edition (1992). HarperCollins Publishers, Inc. 